\title{
Blasenschule hilft Kindern
}

\section{Urotherapie bei kindlicher Inkontinenz und Enuresis}

Die Urotherapie ist in Europa seit mehr als 20 Jahren etabliert und vor allem in den skandinavischen Ländern ein wesentlicher Bestandteil der Behandlung von Kindern mit Inkontinenz, Enuresis und Blasenentleerungsstörungen. Sie umfasst die Diagnostik, Behandlung und Betreuung von Menschen mit funktionellen, organisch bedingten und neurogenen Blasenstö-

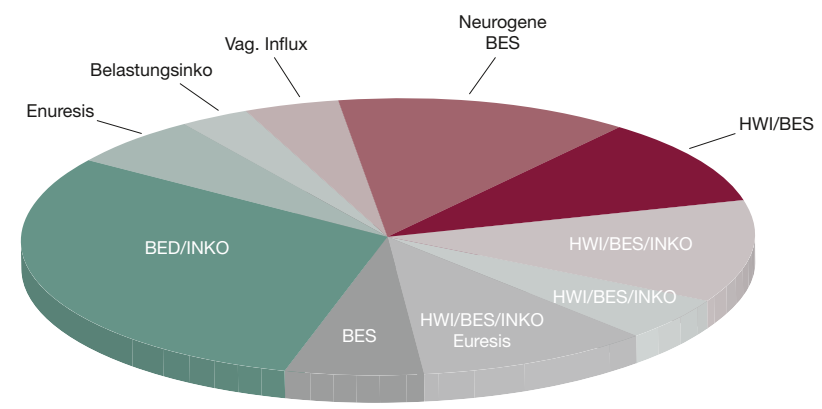

Statistik 2010 rungen bzw. mit Stuhlinkontinenz. Sie beinhaltet sowohl nicht-chirurgische und nicht-pharmakologische Therapien als auch die Unterstützung betroffener Menschen bei chirurgischer und medikamentöser Therapie.

Mittlerweile gibt es in Deutschland einige Zentren, die eine Urotherapie anbieten. Eine deutschsprachige Ausbildung ist seit 2007 am Klinikum Links der Weser in Bremen möglich. Die Ausbildung erfolgt in fünf Modulen, die insgesamt 300 Stunden umfassen. Die Prüfung besteht aus einer schriftlichen Abschlussarbeit sowie einer mündlichen Prüfung. In Österreich bieten momentan zwei Krankenhäuser die Urotherapie an. Eines davon, das Krankenhaus der Barmherzigen Schwestern in Linz, Abteilung für Kinderurologie bietet die Urotherapie in Form ihrer Blasenschule ausschließlich für Kinder an.

Hier werden Kinder betreut und behandelt mit organischer Harninkontinenz:

- kindernephrologische oder kinderurologische Erkrankungen (Diabetes insipitus, Blasenekstrophie, Epispadie, Kloakenfehlbildung, Urethralklappen)

- neurogene Blase angeboren oder erworben (Spina bifida, Sakralagenesie, Tumoren des Rückenmarks, Enzephalitiden, Traumata der Wirbelsäule usw.)

- Hinman-Syndrom: nicht neurogene, neurogene Blasendysfunktion

funktioneller Harninkontinenz:

- HI nachts primär oder sekundär: mono-

DGKS Sieglinde Gratzl, Urotherapeutin KH der Barmherzigen Schwestern Linz symptomatische Enuresis

- HI nachts mit Tagessymptomatik primär oder sekundär: Nicht-monosymptomatische Enuresis

- HI tags primär oder sekundär: Blasendysfunktion

Blasendysfunktion: overactiv bladder (OAB)

- Miktionsaufschub

- Dyskoordinierte Miktion

- Andere Formen: Giggle Inkontinenz, vaginaler Reflux usw.

Bei vielen dieser Krankheitsbilder bestehen Komorbiditäten, wie

- Harnwegsinfektionen mit oder ohne Fieber

- Vesico-ureteraler Reflux

- Obstipation mit oder ohne Stuhlinkontinenz

- psychologische Komorbiditäten: Verhaltensauffälligkeiten, ADHS, familiäre Auffälligkeiten, Lebensereignisse wie z. B.: Geburt eines Geschwisterchens und Tod eines Familienangehörigen oder Trennung der Eltern

- Neuropädiatrische Komorbiditäten: Entwicklungsverzögerung usw.

- Schlafstörungen, wie z. B Schlafapnoesyndrom

\section{Diagnostik}

1. kinderurologische Erstuntersuchung: Basisdiagnostik: Ultraschall, körperliche Untersuchung, Urindiagnostik, Miktionstagebuch, Anamneseerhebung

2. Urotherapeutische Abklärung: 3mal Flow/EMG, Restharnmessungen, ausführliche Anamnese
- Überaktive Blase/Dranginkontinenz:

\section{Therapie}

Die Behandlung erfolgt im Rahmen einer stationären oder tagesstationären Aufnahme. Je nach Krankheitsbild wird jeder Patient einzeln und individuell betreut.

\section{Standard Urotherapie}

- individuelle Betreuung und Aufklärung des Patienten und der betreuenden Personen (Eltern, Erzieher usw.)

- Anatomie/Physiologie/Pathophysiologie (Wie funktioniert die Blase/Niere? Was ist normal, was gestört?)

- Interventionen bzw. Instruktionen (Was kann man tun? Was ist effektiv? Wie soll das Verhalten geändert werden. Trinkmanagement usw.)

- Kontinenzschulung

- Dokumentation

\section{Spezielle Urotherapie}

1. Weckapparat-Therapie

2. Beckenbodentraining

3. Biofeedbacktraining

4. Anleitung Intermittierender Fremd- und Selbstkatheterismus

5. Elektrostimulation

\section{Zusammenfassung}

Die Urotherapie ist in Europa seit über 20 Jahren ein fester Bestandteil der Behandlung von Inkontinenz, Blasenentleerungsstörungen und Enuresis. Unter Urotherapie versteht man die Diagnostik, Behandlung und Betreuung von Menschen mit funktionellen, organisch bedingten und/oder neurogenen Blasenentleerungsstörungen und Stuhlinkontinenz. Die Urotherapie beinhaltet nicht-chirurgische und nicht-pharmakologische Therapien aber auch die Unterstützung betroffener Patienten bei chirurgischen Eingriffen und medikamentöser Therapie.

Die einzige deutschsprachige Ausbildung ist derzeit in Bremen/Deutschland möglich. 